\title{
On the refinements of the Hermite-Hadamard inequality
}

\author{
Sadia Khalid ${ }^{1 *}$ and Josip Pečarić ${ }^{1,2}$
}

\section{"Correspondence:}

saadiakhalid176@gmail.com

${ }^{1}$ Abdus Salam School of

Mathematical Sciences, GC

University, 68-B, New Muslim Town, Lahore 54600, Pakistan

Full list of author information is

available at the end of the article

\begin{abstract}
In this paper, we present some refinements of the classical Hermite-Hadamard integral inequality for convex functions. Further, we give the concept of $n$-exponential convexity and log-convexity of the functions associated with the linear functionals defined by these inequalities and prove monotonicity property of the generalized Cauchy means obtained via these functionals. Finally, we give several examples of the families of functions for which the results can be applied.
\end{abstract}

MSC: 26D15

Keywords: Hermite-Hadamard inequality; refinements; $n$-exponential and logarithmic convexity; mean value theorems

\section{Introduction}

One of the most well-known inequalities in mathematics for convex functions is so called Hermite-Hadamard integral inequality (see [1, p.137])

$$
f\left(\frac{a+b}{2}\right) \leq \frac{1}{b-a} \int_{a}^{b} f(t) d t \leq \frac{f(a)+f(b)}{2}
$$

provided that for an interval $[a, b] \subseteq \mathbb{R}, f:[a, b] \rightarrow \mathbb{R}$ is a convex function. If the function $f$ is concave, then (1) holds in the reverse direction. It gives an estimate from below and above of the mean value of a convex function. These inequalities for convex functions play an important role in nonlinear analysis. In recent years there have been many extensions, generalizations and similar type results of the inequalities in (1) (see [2-4]). These classical inequalities have been improved and generalized in many ways and applied for special means including Stolarsky-type means, logarithmic and $p$-logarithmic means. Also, many interesting applications of Hermite-Hadamard inequality can be found in [1].

In this paper, we present some refinements of the first Hermite-Hadamard integral inequality. Further, we study the $n$-exponential convexity and log-convexity of the functions associated with the linear functionals defined as differences of the left-hand and the righthand sides of these inequalities. We also prove monotonicity property of the generalized Cauchy means obtained via these functionals. Finally, we give several examples of the families of functions for which the obtained results can be applied.

\section{Main results}

We shall start with the following refinement of the first Hermite-Hadamard inequality for differentiable convex functions.

(c) 2012 Khalid and Pečarić; licensee Springer. This is an Open Access article distributed under the terms of the Creative Commons Attribution License (http://creativecommons.org/licenses/by/2.0), which permits unrestricted use, distribution, and reproduction in any medium, provided the original work is properly cited. 
Theorem 2.1 Let $a, b \in \mathbb{R}$ with $a<b$ and $f:[a, b] \rightarrow \mathbb{R}$ be a differentiable convex function. Then the function

$$
H(x)=\frac{1}{b-a}\left[\int_{a}^{x} f(t) d t+(b-x) f(x)\right]-f\left(\frac{2 b x-x^{2}-a^{2}}{2(b-a)}\right)
$$

is increasing on $[a, b]$ and for all $x, y \in[a, b]$ such that $x \leq y$, we have

$$
0 \leq H(x) \leq H(y) \leq \frac{1}{b-a} \int_{a}^{b} f(t) d t-f\left(\frac{a+b}{2}\right) .
$$

Proof We have

$$
H^{\prime}(x)=\frac{b-x}{b-a}\left[f^{\prime}(x)-f^{\prime}\left(\frac{2 b x-x^{2}-a^{2}}{2(b-a)}\right)\right]
$$

where $\frac{b-x}{b-a} \geq 0$, as $a<b$ and $x \in[a, b]$. If we prove that

$$
f^{\prime}(x)-f^{\prime}\left(\frac{2 b x-x^{2}-a^{2}}{2(b-a)}\right) \geq 0
$$

then $H(x)$ will be increasing on $[a, b]$.

For $x \geq a$, we have $x-\frac{2 b x-x^{2}-a^{2}}{2(b-a)} \geq 0$. Since $f$ is a differentiable convex function defined on $[a, b], f^{\prime}$ is increasing on $[a, b]$, and so (4) holds, which in turn implies that $H^{\prime}(x) \geq 0$, showing that $H(x)$ is increasing on $[a, b]$.

Now, as $H(x)$ is increasing on $[a, b]$, for any $x, y \in[a, b]$ such that $a \leq x \leq y \leq b$, we have

$$
H(a) \leq H(x) \leq H(y) \leq H(b) .
$$

At $x=a$ and at $x=b$, (2) gives $H(a)=0$ and $H(b)=\frac{1}{b-a} \int_{a}^{b} f(t) d t-f\left(\frac{a+b}{2}\right)$ respectively. By using these values of $H(a)$ and $H(b)$ in (5), we have (3).

Remark 2.2 If $f$ is strictly convex, then $H(x)$ is strictly increasing on $[a, b)$ and strict inequalities hold in (3).

The second main result is another refinement of the first Hermite-Hadamard inequality for differentiable convex functions.

Theorem 2.3 Let $a, b \in \mathbb{R}$ with $a<b$ and $f:[a, b] \rightarrow \mathbb{R}$ be a differentiable convex function. Then the function

$$
\bar{H}(x)=\frac{1}{b-a}\left[\int_{x}^{b} f(t) d t+(x-a) f(x)\right]-f\left(\frac{x^{2}+b^{2}-2 a x}{2(b-a)}\right)
$$

is decreasing on $[a, b]$ and for any $x, y \in[a, b]$ such that $x \leq y$, we have

$$
0 \leq \bar{H}(y) \leq \bar{H}(x) \leq \frac{1}{b-a} \int_{a}^{b} f(t) d t-f\left(\frac{a+b}{2}\right) .
$$


Proof We have

$$
\bar{H}^{\prime}(x)=\frac{x-a}{b-a}\left[f^{\prime}(x)-f^{\prime}\left(\frac{x^{2}+b^{2}-2 a x}{2(b-a)}\right)\right],
$$

where $\frac{x-a}{b-a} \geq 0$, as $a<b$ and $x \in[a, b]$. If we prove that

$$
f^{\prime}(x)-f^{\prime}\left(\frac{x^{2}+b^{2}-2 a x}{2(b-a)}\right) \leq 0
$$

then $\bar{H}(x)$ will be decreasing on $[a, b]$.

For $x \leq b$, we have $\frac{x^{2}+b^{2}-2 a x}{2(b-a)}-x \geq 0$. Since $f$ is a differentiable convex function defined on $[a, b], f^{\prime}$ is increasing on $[a, b]$, and so (8) holds, which in turn implies that $\bar{H}^{\prime}(x) \leq 0$, showing that $\bar{H}(x)$ is decreasing on $[a, b]$.

Now, as $\bar{H}(x)$ is decreasing on $[a, b]$, for any $x, y \in[a, b]$ such that $a \leq x \leq y \leq b$, we have

$$
\bar{H}(b) \leq \bar{H}(y) \leq \bar{H}(x) \leq \bar{H}(a) .
$$

At $x=a$ and at $x=b$, (6) gives $\bar{H}(a)=\frac{1}{b-a} \int_{a}^{b} f(t) d t-f\left(\frac{a+b}{2}\right)$ and $\bar{H}(b)=0$ respectively. By using these values of $\bar{H}(a)$ and $\bar{H}(b)$ in (9), we have (7).

Remark 2.4 If $f$ is strictly convex, then $\bar{H}(x)$ is strictly decreasing on $(a, b]$ and strict inequalities hold in (7).

Let us observe the inequalities (3) and (7). Motivated by them, we define two functionals

$$
\begin{array}{ll}
\Phi_{1}(x, y ; f)=H(y)-H(x), & x \leq y, \\
\Phi_{2}(x, y ; f)=\bar{H}(x)-\bar{H}(y), & x \leq y,
\end{array}
$$

where $x, y \in[a, b]$ and the functions $H$ and $\bar{H}$ are as in (2) and (6) respectively. If $f$ is a differentiable convex function defined on $[a, b]$, then Theorems 2.1 and 2.3 imply that $\Phi_{i}(x, y ; f) \geq 0, i=1,2$. Now, we give mean value theorems for the functionals $\Phi_{i}, i=1,2$.

Theorem 2.5 Let $f \in C^{2}[a, b]$ and $x, y \in[a, b]$ be such that $x \leq y$. Let $\Phi_{1}$ and $\Phi_{2}$ be linear functionals defined as in (10) and (11). Then there exists $\xi_{1}, \xi_{2} \in[a, b]$ such that

$$
\Phi_{i}(x, y ; f)=\frac{f^{\prime \prime}\left(\xi_{i}\right)}{2} \Phi_{i}\left(x, y ; f_{0}\right), \quad i=1,2,
$$

where $f_{0}(z)=z^{2}$.

Proof Analogous to the proof of Theorem 2.4 in [5].

Theorem 2.6 Let $f, g \in C^{2}[a, b]$ and $x, y \in[a, b]$ be such that $x \leq y$, where $g^{\prime \prime}(x) \neq 0$ for every $x \in[a, b]$. Let $\Phi_{1}$ and $\Phi_{2}$ be linear functionals defined as in (10) and (11). If $\Phi_{1}$ and $\Phi_{2}$ are positive, then there exists $\xi_{1}, \xi_{2} \in[a, b]$ such that

$$
\frac{\Phi_{i}(x, y ; f)}{\Phi_{i}(x, y ; g)}=\frac{f^{\prime \prime}\left(\xi_{i}\right)}{g^{\prime \prime}\left(\xi_{i}\right)}, \quad i=1,2 .
$$


Proof Analogous to the proof of Theorem 2.6 in [5].

Remark 2.7 If the inverse of the function $\frac{f^{\prime \prime}}{g^{\prime \prime}}$ exists, then (13) gives

$$
\xi_{i}=\left(\frac{f^{\prime \prime}}{g^{\prime \prime}}\right)^{-1}\left(\frac{\Phi_{i}(x, y ; f)}{\Phi_{i}(x, y ; g)}\right), \quad i=1,2
$$

\section{3 -exponential convexity and log-convexity of the Hermite-Hadamard differences}

We begin this section by recollecting the definitions and properties which are going to be explored here and also some useful characterizations of these properties. Throughout the paper, $I$ is an open interval in $\mathbb{R}$.

Definition 1 A function $h: I \rightarrow \mathbb{R}$ is $n$-exponentially convex in the Jensen sense on $I$ if

$$
\sum_{i, j=1}^{n} \alpha_{i} \alpha_{j} h\left(\frac{x_{i}+x_{j}}{2}\right) \geq 0
$$

holds for every $\alpha_{i} \in \mathbb{R}$ and $x_{i} \in I, i=1, \ldots, n$ (see [5]).

Definition 2 A function $h: I \rightarrow \mathbb{R}$ is $n$-exponentially convex on $I$ if it is $n$-exponentially convex in the Jensen sense and continuous on $I$.

Remark 3.1 From the above definition it is clear that 1-exponentially convex functions in the Jensen sense are nonnegative functions. Also, $n$-exponentially convex functions in the Jensen sense are $k$-exponentially convex functions in the Jensen sense for all $k \in \mathbb{N}, k \leq n$.

By definition of positive semi-definite matrices and some basic linear algebra, we have the following proposition.

Proposition 3.2 If $h$ is n-exponentially convex in the Jensen sense, then the matrix $\left[h\left(\frac{x_{i}+x_{j}}{2}\right)\right]_{i, j=1}^{k}$ is a positive semi-definite matrix for all $k \in \mathbb{N}, k \leq n$. Particularly,

$$
\operatorname{det}\left[h\left(\frac{x_{i}+x_{j}}{2}\right)\right]_{i, j=1}^{k} \geq 0 \quad \text { for every } k \in \mathbb{N}, k \leq n, x_{i} \in I, i=1, \ldots, n
$$

Definition 3 A function $h: I \rightarrow \mathbb{R}$ is exponentially convex in the Jensen sense if it is $n$-exponentially convex in the Jensen sense for all $n \in \mathbb{N}$.

Definition 4 A function $h: I \rightarrow \mathbb{R}$ is exponentially convex if it is exponentially convex in the Jensen sense and continuous.

Lemma 3.3 A function $h: I \rightarrow(0, \infty)$ is log-convex in the Jensen sense, that is, for every $x, y \in I$,

$$
h^{2}\left(\frac{x+y}{2}\right) \leq h(x) h(y)
$$


holds if and only if the relation

$$
\alpha^{2} h(x)+2 \alpha \beta h\left(\frac{x+y}{2}\right)+\beta^{2} h(y) \geq 0
$$

holds for every $\alpha, \beta \in \mathbb{R}$ and $x, y \in I$.

Remark 3.4 It follows that a function is log-convex in the Jensen sense if and only if it is 2-exponentially convex in the Jensen sense. Also, by using the basic convexity theory, a function is log-convex if and only if it is 2-exponentially convex.

The following result will be useful further (see [1, p.2]).

Lemma 3.5 If $f$ is a convex function defined on an interval I and $x_{1} \leq y_{1}, x_{2} \leq y_{2}, x_{1} \neq x_{2}$, $y_{1} \neq y_{2}$, then the following inequality is valid

$$
\frac{f\left(x_{2}\right)-f\left(x_{1}\right)}{x_{2}-x_{1}} \leq \frac{f\left(y_{2}\right)-f\left(y_{1}\right)}{y_{2}-y_{1}} .
$$

If the function $f$ is concave, the inequality reverses.

Definition 5 The second order divided difference of a function $f:[a, b] \rightarrow \mathbb{R}$ at mutually distinct points $y_{0}, y_{1}, y_{2} \in[a, b]$ is defined recursively by

$$
\begin{aligned}
& {\left[y_{i}, y_{i+1} ; f\right]=\frac{f\left(y_{i+1}\right)-f\left(y_{i}\right)}{y_{i+1}-y_{i}}, \quad i=0,1,} \\
& {\left[y_{0}, y_{1}, y_{2} ; f\right]=\frac{\left[y_{1}, y_{2} ; f\right]-\left[y_{0}, y_{1} ; f\right]}{y_{2}-y_{0}} .}
\end{aligned}
$$

Remark 3.6 The value $\left[y_{0}, y_{1}, y_{2} ; f\right]$ is independent of the order of the points $y_{0}, y_{1}$ and $y_{2}$. This definition may be extended to include the case in which some or all the points coincide (see $\left[1\right.$, p.16]). Namely, taking the limit $y_{1} \rightarrow y_{0}$ in (15), we get

$$
\lim _{y_{1} \rightarrow y_{0}}\left[y_{0}, y_{1}, y_{2} ; f\right]=\left[y_{0}, y_{0}, y_{2} ; f\right]=\frac{f\left(y_{2}\right)-f\left(y_{0}\right)-f^{\prime}\left(y_{0}\right)\left(y_{2}-y_{0}\right)}{\left(y_{2}-y_{0}\right)^{2}}, \quad y_{2} \neq y_{0},
$$

provided that $f^{\prime}$ exists; and furthermore, taking the limits $y_{i} \rightarrow y_{0}, i=1,2$, in (15), we get

$$
\lim _{y_{2} \rightarrow y_{0}} \lim _{y_{1} \rightarrow y_{0}}\left[y_{0}, y_{1}, y_{2} ; f\right]=\left[y_{0}, y_{0}, y_{0} ; f\right]=\frac{f^{\prime \prime}\left(y_{0}\right)}{2},
$$

provided that $f^{\prime \prime}$ exists.

The following definition of a real valued convex function is characterized by second order divided difference (see [1, p.15]).

Definition 6 A function $f:[a, b] \rightarrow \mathbb{R}$ is said to be convex if and only if for all choices of three distinct points $y_{0}, y_{1}, y_{2} \in[a, b],\left[y_{0}, y_{1}, y_{2} ; f\right] \geq 0$.

Next, we study the $n$-exponential convexity and log-convexity of the functions associated with the linear functionals $\Phi_{i}(i=1,2)$ defined in (10) and (11). 
Theorem 3.7 Let $\Omega=\left\{f_{s}: s \in I \subseteq \mathbb{R}\right\}$ be a family of differentiable functions defined on $[a, b]$ such that the function $s \mapsto\left[y_{0}, y_{1}, y_{2} ; f_{s}\right]$ is n-exponentially convex in the Jensen sense on I for every three mutually distinct points $y_{0}, y_{1}, y_{2} \in[a, b]$. Let $\Phi_{i}(i=1,2)$ be linear functionals defined as in (10) and (11). Then the following statements hold.

(i) The function $s \mapsto \Phi_{i}\left(x, y ; f_{s}\right)$ is n-exponentially convex in the Jensen sense on $I$.

(ii) If the function $s \mapsto \Phi_{i}\left(x, y ; f_{s}\right)$ is continuous on $I$, then it is n-exponentially convex on $I$.

Proof The idea of the proof is the same as in [5].

(i) Let $\alpha_{j} \in \mathbb{R}(j=1, \ldots, n)$ and consider the function

$$
g(y)=\sum_{j, k=1}^{n} \alpha_{j} \alpha_{k} \frac{f_{s_{j}+s_{k}}}{2}(y)
$$

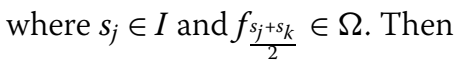

$$
\left[y_{0}, y_{1}, y_{2} ; g\right]=\sum_{j, k=1}^{n} \alpha_{j} \alpha_{k}\left[y_{0}, y_{1}, y_{2} ; \frac{f_{s_{j}+s_{k}}}{2}\right]
$$

and since $\left[y_{0}, y_{1}, y_{2} ; f_{\frac{s_{j}+s_{k}}{2}}\right]$ is $n$-exponentially convex in the Jensen sense on $I$ by assumption, it follows that

$$
\left[y_{0}, y_{1}, y_{2} ; g\right]=\sum_{j, k=1}^{n} \alpha_{j} \alpha_{k}\left[y_{0}, y_{1}, y_{2} ; \frac{f_{s_{j}+s_{k}}}{2}\right] \geq 0 .
$$

And so by using Definition 6, we conclude that $g$ is a convex function. Hence

$$
\Phi_{i}(x, y ; g) \geq 0, \quad i=1,2
$$

which is equivalent to

$$
\sum_{j, k=1}^{n} \alpha_{j} \alpha_{k} \Phi_{i}\left(x, y ; \frac{s_{j+s_{k}}}{2}\right) \geq 0, \quad i=1,2,
$$

and so we conclude that the function $s \mapsto \Phi_{i}\left(x, y ; f_{s}\right)$ is $n$-exponentially convex in the Jensen sense on $I$.

(ii) If the function $s \mapsto \Phi_{i}\left(x, y ; f_{s}\right)$ is continuous on $I$, then from (i) and by Definition 2 it follows that it is $n$-exponentially convex on $I$.

Corollary 3.8 Let $\Omega=\left\{f_{s}: s \in I \subseteq \mathbb{R}\right\}$ be a family of differentiable functions defined on $[a, b]$ such that the function $s \mapsto\left[y_{0}, y_{1}, y_{2} ; f_{s}\right]$ is exponentially convex in the Jensen sense on I for every three mutually distinct points $y_{0}, y_{1}, y_{2} \in[a, b]$. Let $\Phi_{i}(i=1,2)$ be linear functionals defined as in (10) and (11). Then the following statements hold.

(i) The function $s \mapsto \Phi_{i}\left(x, y ; f_{s}\right)$ is exponentially convex in the Jensen sense on $I$.

(ii) If the function $s \mapsto \Phi_{i}\left(x, y ; f_{s}\right)$ is continuous on $I$, then it is exponentially convex on $I$. 
Corollary 3.9 Let $\Omega=\left\{f_{s}: s \in I \subseteq \mathbb{R}\right\}$ be a family of differentiable functions defined on $[a, b]$ such that the function $s \mapsto\left[y_{0}, y_{1}, y_{2} ; f_{s}\right]$ is 2-exponentially convex in the Jensen sense on I for every three mutually distinct points $y_{0}, y_{1}, y_{2} \in[a, b]$. Let $\Phi_{i}(i=1,2)$ be linear functionals defined as in (10) and (11). Further, assume $\Phi_{i}\left(x, y ; f_{s}\right)(i=1,2)$ is strictly positive for $f_{s} \in \Omega$. Then the following statements hold:

(i) If the function $s \mapsto \Phi_{i}\left(x, y ; f_{s}\right)$ is continuous on $I$, then it is 2-exponentially convex on $I$ and so, it is log-convex.

(ii) If the function $s \mapsto \Phi_{i}\left(x, y ; f_{s}\right)$ is differentiable on I, then for every $s, q, u, v \in I$ such that $s \leq u$ and $q \leq v$, we have

$$
\mu_{s, q}\left(x, y, \Phi_{i}, \Omega\right) \leq \mu_{u, v}\left(x, y, \Phi_{i}, \Omega\right), \quad i=1,2
$$

where

$$
\mu_{s, q}\left(x, y, \Phi_{i}, \Omega\right)= \begin{cases}\left(\frac{\Phi_{i}\left(x, y ; f_{s}\right)}{\Phi_{i}\left(x, y ; f_{q}\right)}\right)^{\frac{1}{s-q}}, & s \neq q, \\ \exp \left(\frac{\frac{d}{d s} \Phi_{i}\left(x, y ; f_{s}\right)}{\Phi_{i}\left(x, y ; f_{s}\right)}\right), & s=q,\end{cases}
$$

for $f_{s}, f_{q} \in \Omega$.

Proof The idea of the proof is the same as in [5].

(i) The claim is an immediate consequence of Theorem 3.7 and Remark 3.4.

(ii) Since by (i) the function $s \mapsto \Phi_{i}\left(x, y\right.$; $\left.f_{s}\right)$ is log-convex on $I$, that is, the function $s \mapsto \log \Phi_{i}\left(x, y ; f_{s}\right)$ is convex on $I$. Applying Lemma 3.5 with setting $f(z)=\log \Phi_{i}\left(x, y ; f_{z}\right)(i=1,2)$, we get

$$
\frac{\log \Phi_{i}\left(x, y ; f_{s}\right)-\log \Phi_{i}\left(x, y ; f_{q}\right)}{s-q} \leq \frac{\log \Phi_{i}\left(x, y ; f_{u}\right)-\log \Phi_{i}\left(x, y ; f_{v}\right)}{u-v}
$$

for $s \leq u, q \leq v, s \neq q, u \neq v$; and therefore conclude that

$$
\mu_{s, q}\left(x, y, \Phi_{i}, \Omega\right) \leq \mu_{u, v}\left(x, y, \Phi_{i}, \Omega\right), \quad i=1,2
$$

If $s=q$, we consider the limit when $q \rightarrow s$ in (18) and conclude that

$$
\mu_{s, s}\left(x, y, \Phi_{i}, \Omega\right) \leq \mu_{u, v}\left(x, y, \Phi_{i}, \Omega\right), \quad i=1,2 .
$$

The case $u=v$ can be treated similarly.

Remark 3.10 Note that the results from Theorem 3.7, Corollary 3.8 and Corollary 3.9 still hold when two of the points $y_{0}, y_{1}, y_{2} \in[a, b]$ coincide, say $y_{1}=y_{0}$, for a family of differentiable functions $f_{s}$ such that the function $s \mapsto\left[y_{0}, y_{1}, y_{2} ; f_{s}\right]$ is $n$-exponentially convex in the Jensen sense (exponentially convex in the Jensen sense, log-convex in the Jensen sense on $I$ ); and furthermore, they still hold when all three points coincide for a family of twice differentiable functions with the same property. The proofs are obtained by recalling Remark 3.6 and by using suitable characterizations of convexity. 


\section{Examples}

In this section, we present several families of functions which fulfill the conditions of Theorem 3.7, Corollary 3.8 and Corollary 3.9 (Remark 3.10). In this way, we can construct large families of functions which are exponentially convex.

Example 4.1 Consider the family of functions

$$
\Omega_{1}=\left\{g_{s}: \mathbb{R} \rightarrow[0, \infty): s \in \mathbb{R}\right\}
$$

defined by

$$
g_{s}(x)= \begin{cases}\frac{1}{s^{2}} e^{s x}, & s \neq 0, \\ \frac{1}{2} x^{2}, & s=0 .\end{cases}
$$

We have $\frac{d^{2}}{d x^{2}} g_{s}(x)=e^{s x}>0$ which shows that $g_{s}$ is convex on $\mathbb{R}$ for every $s \in \mathbb{R}$ and $s \mapsto$ $\frac{d^{2}}{d x^{2}} g_{s}(x)$ is exponentially convex by Example 4.1 given in [6]. From [6], we then also have that $s \mapsto\left[y_{0}, y_{1}, y_{2} ; g_{s}\right]$ is exponentially convex and so $s \mapsto\left[y_{0}, y_{1}, y_{2} ; g_{s}\right]$ is exponentially convex in Jensen sense. Now, by using Corollary 3.8, we have $s \mapsto \Phi_{i}\left(x, y ; g_{s}\right)(i=1,2)$ are exponentially convex in Jensen sense. Since these mappings are continuous (although the mapping $s \mapsto g_{s}$ is not continuous for $\left.s=0\right), s \mapsto \Phi_{i}\left(x, y ; g_{s}\right)(i=1,2)$ are exponentially convex.

For this family of functions, by taking $\Omega=\Omega_{1}$ in (17), $\Xi_{s, q ; 1}^{i}:=\mu_{s, q}\left(x, y, \Phi_{i}, \Omega_{1}\right)(i=1,2)$ are of the form

$$
\begin{aligned}
& \Xi_{s, q ; 1}^{1}=\left(\frac{q^{2}}{s^{2}} \cdot \frac{\frac{e^{s y}-e^{s x}}{s}+e^{s y}(b-y)-e^{s x}(b-x)+(b-a)\left(e^{s \hat{x}}-e^{s \hat{y}}\right)}{\frac{e^{q y}-e^{q x}}{q}+e^{q y}(b-y)-e^{q x}(b-x)+(b-a)\left(e^{q \hat{x}}-e^{q \hat{y}}\right)}\right)^{\frac{1}{s-q}}, \quad s \neq q \neq 0,
\end{aligned}
$$

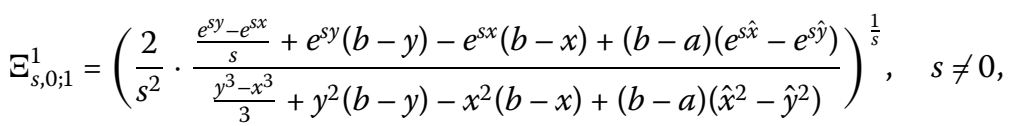

$$
\begin{aligned}
& \Xi_{s, s ; 1}^{1}=\exp \left(\frac{1}{s} \cdot \frac{e^{s x}\left(\frac{3}{s}-x-(b-x)(s x-2)\right)+e^{s y}\left(y-\frac{3}{s}+(b-y)(s y-2)\right)}{\frac{+(b-a)\left(e^{s \hat{x}}(s \hat{x}-2)-e^{s \hat{y}}(s \hat{y}-2)\right)}{s}+e^{s y}(b-y)-e^{s x}(b-x)+(b-a)\left(e^{s \hat{x}}-e^{s \hat{y}}\right)}\right), \quad s \neq 0, \\
& \Xi_{0,0 ; 1}^{1}=\exp \left(\frac{1}{3} \cdot \frac{\frac{y^{4}-x^{4}}{4}+y^{3}(b-y)-x^{3}(b-x)+(b-a)\left(\hat{x}^{3}-\hat{y}^{3}\right)}{\frac{y^{3}-x^{3}}{3}+y^{2}(b-y)-x^{2}(b-x)+(b-a)\left(\hat{x}^{2}-\hat{y}^{2}\right)}\right), \\
& \Xi_{s, q ; 1}^{2}=\left(\frac{q^{2}}{s^{2}} \cdot \frac{\frac{e^{s y}-e^{s x}}{s}+e^{s x}(x-a)-e^{s y}(y-a)+(b-a)\left(e^{s \tilde{y}}-e^{s \tilde{x}}\right)}{\frac{e^{q y}-e^{q x}}{q}+e^{q x}(x-a)-e^{q y}(y-a)+(b-a)\left(e^{q \tilde{y}}-e^{q \tilde{x}}\right)}\right)^{\frac{1}{s-q}}, \quad s \neq q \neq 0, \\
& \Xi_{s, 0 ; 1}^{2}=\left(\frac{2}{s^{2}} \cdot \frac{\frac{e^{s y}-e^{s x}}{s}+e^{s x}(x-a)-e^{s y}(y-a)+(b-a)\left(e^{s \tilde{y}}-e^{s \tilde{x}}\right)}{\frac{y^{3}-x^{3}}{3}+x^{2}(x-a)-y^{2}(y-a)+(b-a)\left(\tilde{y}^{2}-\tilde{x}^{2}\right)}\right)^{\frac{1}{s}}, \quad s \neq 0, \\
& \Xi_{s, s ; 1}^{2}=\exp \left(\frac{1}{s} \cdot \frac{e^{s x}\left(\frac{3}{s}-x+(x-a)(s x-2)\right)+e^{s y}\left(y-\frac{3}{s}-(y-a)(s y-2)\right)}{\frac{+(b-a)\left(e^{s \tilde{y}}(s \tilde{y}-2)-e^{s \tilde{x}}(s \tilde{x}-2)\right)}{e^{s y}-e^{s x}}+e^{s x}(x-a)-e^{s y}(y-a)+(b-a)\left(e^{s \tilde{y}}-e^{s \tilde{x}}\right)}\right), \quad s \neq 0, \\
& \Xi_{0,0 ; 1}^{2}=\exp \left(\frac{1}{3} \cdot \frac{\frac{y^{4}-x^{4}}{4}+x^{3}(x-a)-y^{3}(y-a)+(b-a)\left(\tilde{y}^{3}-\tilde{x}^{3}\right)}{\frac{y^{3}-x^{3}}{3}+x^{2}(x-a)-y^{2}(y-a)+(b-a)\left(\tilde{y}^{2}-\tilde{x}^{2}\right)}\right),
\end{aligned}
$$


where

$$
\begin{array}{ll}
\hat{x}=\frac{2 b x-x^{2}-a^{2}}{2(b-a)}, & \hat{y}=\frac{2 b y-y^{2}-a^{2}}{2(b-a)}, \\
\tilde{x}=\frac{x^{2}+b^{2}-2 a x}{2(b-a)}, & \tilde{y}=\frac{y^{2}+b^{2}-2 a y}{2(b-a)} .
\end{array}
$$

By using Theorem 2.6, it can be seen that

$$
M_{s, q}\left(x, \Phi_{i}, \Omega_{1}\right)=\log \mu_{s, q}\left(x, \Phi_{i}, \Omega_{1}\right), \quad i=1,2,
$$

satisfy $\min \{a, b\} \leq M_{s, q}\left(x, \Phi_{i}, \Omega_{1}\right) \leq \max \{a, b\}$, showing that $M_{s, q}\left(x, \Phi_{i}, \Omega_{1}\right)(i=1,2)$ are means.

Example 4.2 Consider the family of functions

$$
\Omega_{2}=\left\{f_{s}:(0, \infty) \rightarrow \mathbb{R}: s \in \mathbb{R}\right\}
$$

defined by

$$
f_{s}(x)= \begin{cases}\frac{x^{s}}{s(s-1)}, & s \neq 0,1, \\ -\ln x, & s=0, \\ x \ln x, & s=1 .\end{cases}
$$

Here, $\frac{d^{2}}{d x^{2}} f_{s}(x)=x^{s-2}=e^{(s-2) \ln x}>0$ which shows that $f_{s}$ is convex for $x>0$ and $s \mapsto \frac{d^{2}}{d x^{2}} f_{s}(x)$ is exponentially convex by Example 4.2 given in [6]. From [6], we have $s \mapsto\left[y_{0}, y_{1}, y_{2} ; f_{s}\right.$ ] is exponentially convex. Arguing as in Example 4.1, we have $s \mapsto \Phi_{i}\left(x, y ; f_{s}\right)(i=1,2)$ are exponentially convex.

By taking $\Omega=\Omega_{2}$ in (17), $\Xi_{s, q ; 2}^{i}:=\mu_{s, q}\left(x, y, \Phi_{i}, \Omega_{2}\right)(i=1,2)$ for $x, y>0$, where $x, y \in[a, b]$, are of the form

$$
\begin{aligned}
& \Xi_{s, q ; 2}^{1}=\left(\frac{q(q-1)}{s(s-1)} \cdot \frac{\frac{y^{s+1}-x^{s+1}}{s+1}+y^{s}(b-y)-x^{s}(b-x)+(b-a)\left(\hat{x}^{s}-\hat{y}^{s}\right)}{\frac{y^{q+1}-x^{q+1}}{q+1}+y^{q}(b-y)-x^{q}(b-x)+(b-a)\left(\hat{x}^{q}-\hat{y}^{q}\right)}\right)^{\frac{1}{s-q}}, \\
& s \neq q \neq-1,0,1, \\
& \Xi_{s, 0 ; 2}^{1}=\left(\frac{1}{s(s-1)} \cdot \frac{\frac{y^{s+1}-x^{s+1}}{s+1}+y^{s}(b-y)-x^{s}(b-x)+(b-a)\left(\hat{x}^{s}-\hat{y}^{s}\right)}{y-x+b(\ln x-\ln y)+(b-a)(\ln \hat{y}-\ln \hat{x})}\right)^{\frac{1}{s}}, \quad s \neq-1,0,1, \\
& \Xi_{s, 1 ; 2}^{1}=\left(\frac{4}{s(s-1)} \cdot \frac{\frac{y^{s+1}-x^{s+1}}{s+1}+y^{s}(b-y)-x^{s}(b-x)+(b-a)\left(\hat{x}^{s}-\hat{y}^{s}\right)}{x^{2}-y^{2}+2[y \ln y(2 b-y)-x \ln x(2 b-x)]}\right)^{\frac{1}{s-1}}, \quad s \neq-1,0,1, \\
& \Xi_{0,1 ; 2}^{1}=\frac{x^{2}-y^{2}+2[y \ln y(2 b-y)-x \ln x(2 b-x)]+4(b-a)(\hat{x} \ln \hat{x}-\hat{y} \ln \hat{y})}{4[y-x+b(\ln x-\ln y)+(b-a)(\ln \hat{y}-\ln \hat{x})]}, \\
& \frac{1}{b-a}\left[\frac{y^{s+1} \ln y-x^{s+1} \ln x}{s+1}+\frac{\left(1-3 s^{2}\right)\left(y^{s+1}-x^{s+1}\right)}{s(s-1)(s+1)^{2}}+y^{s}(b-y)\left(\frac{1-2 s}{s(s-1)}+\ln y\right)\right. \\
& \Xi_{s, s ; 2}^{1}=\exp \left(\frac{\left.-x^{s}(b-x)\left(\frac{1-2 s}{s(s-1)}+\ln x\right)\right]+\frac{1-2 s}{s(s-1)}\left(\hat{x}^{s}-\hat{y}^{s}\right)+\hat{x}^{s} \ln \hat{x}-\hat{y}^{s} \ln \hat{y}}{\frac{1}{b-a}\left(\frac{y^{s+1}-x^{s+1}}{s+1}+y^{s}(b-y)-x^{s}(b-x)+(b-a)\left(\hat{x}^{s}-\hat{y}^{s}\right)\right)}\right), \quad s \neq-1,0,1 \text {, }
\end{aligned}
$$




$$
\begin{aligned}
& \Xi_{0,0 ; 2}^{1}=\exp \left(\frac{\begin{array}{c}
\ln x[x \ln x+(b-x)(2+\ln x)]-\ln y[y \ln y-(b-y)(2+\ln y)] \\
+(b-a)[\ln \hat{y}(2+\ln \hat{y})-\ln \hat{x}(2+\ln \hat{x})]
\end{array}}{2[y-x+b(\ln x-\ln y)+(b-a)(\ln \hat{y}-\ln \hat{x})]}\right), \\
& \Xi_{1,1 ; 2}^{1}=\exp \left(\begin{array}{c}
2[y \ln y\{y \ln y-3 y+2(b-y)(\ln y-2)\}-x \ln x(x \ln x-3 x)]+3\left(y^{2}-x^{2}\right) \\
2\left[x^{2}-y^{2}+2\{y \ln y(2 b-y)-x \ln x(2 b-x)\}+4(b-a)(\hat{x} \ln \hat{x}-\hat{y} \ln \hat{y})\right]
\end{array}\right), \\
& \Xi_{s, q ; 2}^{2}=\left(\frac{q(q-1)}{s(s-1)} \cdot \frac{\frac{y^{s+1}-x^{s+1}}{s+1}+x^{s}(x-a)-y^{s}(y-a)+(b-a)\left(\tilde{y}^{s}-\tilde{x}^{s}\right)}{\frac{y^{q+1}-x^{q+1}}{q+1}+x^{q}(x-a)-y^{q}(y-a)+(b-a)\left(\tilde{y}^{q}-\tilde{x}^{q}\right)}\right)^{\frac{1}{s-q}}, \\
& s \neq q \neq-1,0,1 \text {, }
\end{aligned}
$$$$
\Xi_{s, 0 ; 2}^{2}=\left(\frac{1}{s(s-1)} \cdot \frac{\frac{y^{s+1}-x^{s+1}}{s+1}+x^{s}(x-a)-y^{s}(y-a)+(b-a)\left(\tilde{y}^{s}-\tilde{x}^{s}\right)}{y-x+a(\ln x-\ln y)+(b-a)(\ln \tilde{x}-\ln \tilde{y})}\right)^{\frac{1}{s}}, \quad s \neq-1,0,1,
$$$$
\Xi_{s, 1 ; 2}^{2}=\left(\frac{4}{s(s-1)} \cdot \frac{\frac{y^{s+1}-x^{s+1}}{s+1}+x^{s}(x-a)-y^{s}(y-a)+(b-a)\left(\tilde{y}^{s}-\tilde{x}^{s}\right)}{x^{2}-y^{2}+2[y \ln y(2 a-y)-x \ln x(2 a-x)]}\right)^{\frac{1}{s-1}}, \quad s \neq-1,0,1,
$$$$
\Xi_{0,1 ; 2}^{2}=\frac{x^{2}-y^{2}+2[y \ln y(2 a-y)-x \ln x(2 a-x)]+4(b-a)(\tilde{y} \ln \tilde{y}-\tilde{x} \ln \tilde{x})}{4[y-x+a(\ln x-\ln y)+(b-a)(\ln \tilde{x}-\ln \tilde{y})]},
$$$$
\Xi_{s, s ; 2}^{2}=\exp \left(\frac{\frac{1}{b-a}\left[\frac{y^{s+1} \ln y-x^{s+1} \ln x}{s+1}+\frac{\left(1-3 s^{2}\right)\left(y^{s+1}-x^{s+1}\right)}{s(s-1)(s+1)^{2}}+x^{s}(x-a)\left(\frac{1-2 s}{s(s-1)}+\ln x\right)\right.}{\frac{\left.-y^{s}(y-a)\left(\frac{1-2 s}{s(s-1)}+\ln y\right)\right]+\frac{1-2 s}{s(s-1)}\left(\tilde{y}^{s}-\tilde{x}^{s}\right)+\tilde{y}^{s} \ln \tilde{y}-\tilde{x}^{s} \ln \tilde{x}}{b-a}\left(\frac{y^{s+1}-x^{s+1}}{s+1}+x^{s}(x-a)-y^{s}(y-a)+(b-a)\left(\tilde{y}^{s}-\tilde{x}^{s}\right)\right)}\right), \quad s \neq-1,0,1,
$$$$
\Xi_{0,0 ; 2}^{2}=\exp \left(\frac{\begin{array}{c}
\ln x[x \ln x-(x-a)(2+\ln x)]-\ln y[y \ln y-(y-a)(2+\ln y)] \\
+(b-a)[\ln \tilde{x}(2+\ln \tilde{x})-\ln \tilde{y}(2+\ln \tilde{y})]
\end{array}}{2[y-x+a(\ln x-\ln y)+(b-a)(\ln \tilde{x}-\ln \tilde{y})]}\right),
$$

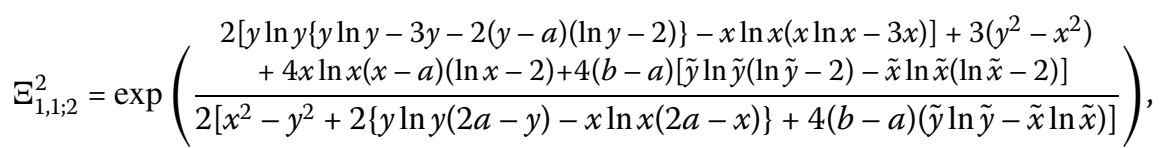

where $\hat{x}, \hat{y}, \tilde{x}$ and $\tilde{y}$ are the same as in (19). If $\Phi_{i}(i=1,2)$ are positive, then Theorem 2.6 applied for $f=f_{s} \in \Omega_{2}$ and $g=f_{q} \in \Omega_{2}$ yields that there exists $\xi_{i} \in[a, b]$ such that

$$
\xi_{i}^{s-q}=\frac{\Phi_{i}\left(x, y ; f_{s}\right)}{\Phi_{i}\left(x, y ; f_{q}\right)}, \quad i=1,2
$$

Since the functions $\xi_{i} \mapsto \xi_{i}^{s-q}(i=1,2)$ are invertible for $s \neq q$, we then have

$$
\min \{a, b\} \leq\left(\frac{\Phi_{i}\left(x, y ; f_{s}\right)}{\Phi_{i}\left(x, y ; f_{q}\right)}\right)^{\frac{1}{s-q}} \leq \max \{a, b\}, \quad i=1,2
$$

which, together with the fact that $\mu_{s, q}\left(x, y, \Phi_{i}, \Omega_{2}\right)(i=1,2)$ are continuous, symmetric and monotonous (by (16)), shows that $\mu_{s, q}\left(x, y, \Phi_{i}, \Omega_{2}\right)(i=1,2)$ are means. 
Now, by the substitutions $x \rightarrow x^{t}, y \rightarrow y^{t}, s \rightarrow \frac{s}{t}, q \rightarrow \frac{q}{t}(t \neq 0, s \neq q)$, where $x, y \in[a, b]$, from (20) we get

$$
\min \left\{a^{t}, b^{t}\right\} \leq\left(\frac{\Phi_{i}\left(x^{t}, y^{t} ; f_{s / t}\right.}{\Phi_{i}\left(x^{t}, y^{t} ; f_{q / t}\right.}\right)^{\frac{t}{s-q}} \leq \max \left\{a^{t}, b^{t}\right\}
$$

We define a new mean (for $i=1,2$ ) as follows:

$$
\mu_{s, q ; t}\left(x, y, \Phi_{i}, \Omega_{2}\right)= \begin{cases}\left(\mu_{\frac{s}{t}, \frac{q}{t}}\left(x^{t}, y^{t}, \Phi_{i}, \Omega_{2}\right)\right)^{\frac{1}{t},} & t \neq 0, \\ \mu_{s, q}\left(\ln x, \ln y, \Phi_{i}, \Omega_{1}\right), & t=0 .\end{cases}
$$

These new means are also monotonous. More precisely, for $s, q, u, v \in \mathbb{R}$ such that $s \leq u$, $q \leq v, s \neq q, u \neq v$, we have

$$
\mu_{s, q ; t}\left(x, y, \Phi_{i}, \Omega_{2}\right) \leq \mu_{u, v ; t}\left(x, y, \Phi_{i}, \Omega_{2}\right), \quad i=1,2
$$

We know that

$$
\mu_{\frac{s}{t}, \frac{q}{t}}\left(x^{t}, y^{t}, \Phi_{i}, \Omega_{2}\right) \leq \mu_{\frac{u}{t}, \frac{v}{t}}\left(x^{t}, y^{t}, \Phi_{i}, \Omega_{2}\right), \quad i=1,2,
$$

equivalently

$$
\left(\frac{\Phi_{i}\left(x^{t}, y^{t} ; f_{s / t}\right)}{\Phi_{i}\left(x^{t}, y^{t} ; f_{q / t}\right)}\right)^{\frac{t}{s-q}} \leq\left(\frac{\Phi_{i}\left(x^{t}, y^{t} ; f_{u / t}\right)}{\Phi_{i}\left(x^{t}, y^{t} ; f_{v / t}\right)}\right)^{\frac{t}{u-v}}, \quad i=1,2,
$$

for $s, q, u, v \in I$ such that $s / t \leq u / t, q / t \leq v / t$ and $t \neq 0$, since $\mu_{s, q}\left(x, y, \Phi_{i}, \Omega_{2}\right)(i=1,2)$ are monotonous in both parameters, the claim follows. For $t=0$, we obtain the required result by taking the limit $t \rightarrow 0$.

Remark 4.3 If we make the substitutions $x \rightarrow a, y \rightarrow b, s \rightarrow s-1$ and $t \rightarrow t-1$ in our means $\mu_{s, q}\left(x, y, \Phi_{i}, \Omega_{2}\right)$ and $\mu_{s, q ; t}\left(x, y, \Phi_{i}, \Omega_{2}\right)(i=1,2)$, then the results for the $E_{1}(r, t)$ means and the generalized $E_{1}$ means given in [7] are recaptured. In this way our results for means are the generalizations of the above mentioned means.

Example 4.4 Consider the family of functions

$$
\Omega_{3}=\left\{h_{s}:(0, \infty) \rightarrow(0, \infty): s \in(0, \infty)\right\}
$$

defined by

$$
h_{s}(x)= \begin{cases}\frac{s^{-x}}{\ln ^{2} s}, & s \neq 1, \\ \frac{x^{2}}{2}, & s=1 .\end{cases}
$$

We have $\frac{d^{2}}{d x^{2}} h_{s}(x)=s^{-x}>0$ which shows that $h_{s}$ is convex for all $s>0$. Exponential convexity of $s \mapsto \frac{d^{2}}{d x^{2}} h_{s}(x)=s^{-x}$ on $(0, \infty)$ is given by Example 4.3 in [6]. Arguing as in Example 4.1, we have $s \mapsto \Phi_{i}\left(x, y ; h_{s}\right)(i=1,2)$ are exponentially convex. 
In this case by taking $\Omega=\Omega_{3}$ in (17), $\Xi_{s, q ; 3}^{i}:=\mu_{s, q}\left(x, y, \Phi_{i}, \Omega_{3}\right)(i=1,2)$ for $x, y>0$, where $x, y \in[a, b]$, are of the form

$$
\begin{aligned}
& \Xi_{s, q ; 3}^{1}=\left(\frac{\ln ^{2} q}{\ln ^{2} s} \cdot \frac{\frac{s^{-x}-s^{-y}}{\ln s}+s^{-y}(b-y)-s^{-x}(b-x)+(b-a)\left(s^{-\hat{x}}-s^{-\hat{y}}\right)}{\frac{q^{-x}-q^{-y}}{\ln q}+q^{-y}(b-y)-q^{-x}(b-x)+(b-a)\left(q^{-\hat{x}}-q^{-\hat{y}}\right)}\right)^{\frac{1}{s-q}}, \quad s \neq q \neq 1, \\
& \Xi_{s, 1 ; 3}^{1}=\left(\frac{2}{\ln ^{2} s} \cdot \frac{\frac{s^{-x}-s^{-y}}{\ln s}+s^{-y}(b-y)-s^{-x}(b-x)+(b-a)\left(s^{-\hat{x}}-s^{-\hat{y}}\right)}{\frac{y^{3}-x^{3}}{3}+y^{2}(b-y)-x^{2}(b-x)+(b-a)\left(\hat{x}^{2}-\hat{y}^{2}\right)}\right)^{\frac{1}{s-1}}, \quad s \neq 1, \\
& \Xi_{s, s ; 3}^{1}=\exp \left(\begin{array}{c}
\frac{1}{b-a}\left[y s^{-y}-x s^{-x}+\frac{3}{\ln s}\left(s^{-y}-s^{-x}\right)-s^{-y}(b-y)(2+y \ln s)\right. \\
\frac{\left.+s^{-x}(b-x)(2+x \ln s)\right]-s^{-\hat{x}}(2+\hat{x} \ln s)+s^{-\hat{y}}(2+\hat{y} \ln s)}{b-a}\left(\frac{s^{-x}-s^{-y}}{\ln s}+s^{-y}(b-y)-s^{-x}(b-x)+(b-a)\left(s^{-\hat{x}}-s^{-\hat{y}}\right)\right)
\end{array}\right), \quad s \neq 1, \\
& \Xi_{1,1 ; 3}^{1}=\exp \left(-\frac{1}{3} \cdot \frac{\frac{y^{4}-x^{4}}{4}+y^{3}(b-y)-x^{3}(b-x)+(b-a)\left(\hat{x}^{3}-\hat{y}^{3}\right)}{\frac{y^{3}-x^{3}}{3}+y^{2}(b-y)-x^{2}(b-x)+(b-a)\left(\hat{x}^{2}-\hat{y}^{2}\right)}\right), \\
& \Xi_{s, q ; 3}^{2}=\left(\frac{\ln ^{2} q}{\ln ^{2} s} \cdot \frac{\frac{s^{-x}-s^{-y}}{\ln s}+s^{-x}(x-a)-s^{-y}(y-a)+(b-a)\left(s^{-\tilde{y}}-s^{-\tilde{x}}\right)}{\frac{q^{-x}-q^{-y}}{\ln q}+q^{-x}(x-a)-q^{-y}(y-a)+(b-a)\left(q^{-\tilde{y}}-q^{-\tilde{x}}\right)}\right)^{\frac{1}{s-q}}, \quad s \neq q \neq 1, \\
& \Xi_{s, 1 ; 3}^{2}=\left(\frac{2}{\ln ^{2} s} \cdot \frac{\frac{s^{-x}-s^{-y}}{\ln s}+s^{-x}(x-a)-s^{-y}(y-a)+(b-a)\left(s^{-\tilde{y}}-s^{-\tilde{x}}\right)}{\frac{y^{3}-x^{3}}{3}+x^{2}(x-a)-y^{2}(y-a)+(b-a)\left(\tilde{y}^{2}-\tilde{x}^{2}\right)}\right)^{\frac{1}{s-1}}, \quad s \neq 1, \\
& \Xi_{s, s ; 3}^{2}=\exp \left(\begin{array}{c}
\frac{1}{b-a}\left[y s^{-y}-x s^{-x}+\frac{3}{\ln s}\left(s^{-y}-s^{-x}\right)-s^{-x}(x-a)(2+x \ln s)\right. \\
\frac{\left.+s^{-y}(y-a)(2+y \ln s)\right]-s^{-\tilde{y}}(2+\tilde{y} \ln s)+s^{-\tilde{x}}(2+\tilde{x} \ln s)}{b-a}\left(\frac{s^{-x}-s^{-y}}{\ln s}+s^{-x}(x-a)-s^{-y}(y-a)+(b-a)\left(s^{-\tilde{y}}-s^{-\tilde{x}}\right)\right)
\end{array}\right), \quad s \neq 1, \\
& \Xi_{1,1 ; 3}^{2}=\exp \left(-\frac{1}{3} \cdot \frac{\frac{y^{4}-x^{4}}{4}+x^{3}(x-a)-y^{3}(y-a)+(b-a)\left(\tilde{y}^{3}-\tilde{x}^{3}\right)}{\frac{y^{3}-x^{3}}{3}+x^{2}(x-a)-y^{2}(y-a)+(b-a)\left(\tilde{y}^{2}-\tilde{x}^{2}\right)}\right),
\end{aligned}
$$

where $\hat{x}, \hat{y}, \tilde{x}$ and $\tilde{y}$ are the same as in (19). By using Theorem 2.6, it follows that

$$
M_{s, q}\left(x, \Phi_{i}, \Omega_{3}\right)=-L(s, q) \log \mu_{s, q}\left(x, \Phi_{i}, \Omega_{3}\right), \quad i=1,2,
$$

satisfy $\min \{a, b\} \leq M_{s, q}\left(x, \Phi_{i}, \Omega_{3}\right) \leq \max \{a, b\}$ and so $M_{s, q}\left(x, \Phi_{i}, \Omega_{3}\right)(i=1,2)$ are means, where $L(s, q)$ is a logarithmic mean defined by $L(s, q)=\frac{s-q}{\log s-\log q}, s \neq q, L(s, s)=s$.

Example 4.5 Consider the family of functions

$$
\Omega_{4}=\left\{k_{s}:(0, \infty) \rightarrow(0, \infty): s \in(0, \infty)\right\}
$$

defined by

$$
k_{s}(x)=\frac{e^{-x \sqrt{s}}}{s} .
$$

Here, $\frac{d^{2}}{d x^{2}} k_{s}(x)=e^{-x \sqrt{s}}>0$ which shows that $k_{s}$ is convex for all $s>0$. Exponential convexity of $s \mapsto \frac{d^{2}}{d x^{2}} k_{s}(x)=e^{-x \sqrt{s}}$ on $(0, \infty)$ is given by Example 4.4 in [6]. Arguing as in Example 4.1, we have $s \mapsto \Phi_{i}\left(x, y ; k_{s}\right)(i=1,2)$ are exponentially convex. 
In this case by taking $\Omega=\Omega_{4}$ in (17), $\Xi_{s, q ; 4}^{i}:=\mu_{s, q}\left(x, y, \Phi_{i}, \Omega_{4}\right)(i=1,2)$ for $x, y>0$, where $x, y \in[a, b]$, are of the form

$$
\begin{aligned}
& \Xi_{s, q ; 4}^{1}=\left(\frac{q}{s} \cdot \frac{\frac{e^{-x \sqrt{s}}-e^{-y \sqrt{s}}}{\sqrt{s}}+e^{-y \sqrt{s}}(b-y)-e^{-x \sqrt{s}}(b-x)+(b-a)\left(e^{-\hat{x} \sqrt{s}}-e^{-\hat{y} \sqrt{s}}\right)}{\frac{e^{-x \sqrt{q}}-e^{-y \sqrt{q}}}{\sqrt{q}}+e^{-y \sqrt{q}}(b-y)-e^{-x \sqrt{q}}(b-x)+(b-a)\left(e^{-\hat{x} \sqrt{q}}-e^{-\hat{y} \sqrt{q}}\right)}\right)^{\frac{1}{s-q}}, \\
& s \neq q, \\
& \Xi_{s, s ; 4}^{1}=\exp \left(\frac{y e^{-y \sqrt{s}}-x e^{-x \sqrt{s}}+\frac{3}{\sqrt{s}}\left(e^{-y \sqrt{s}}-e^{-x \sqrt{s}}\right)-e^{-y \sqrt{s}}(b-y)(2+y \sqrt{s})}{2 s\left(\frac{e^{-x \sqrt{s}}-e^{-y \sqrt{s}}}{\sqrt{s}}+e^{-y \sqrt{s}}(b-y)-e^{-x \sqrt{s}}(b-x)+(b-a)\left(e^{-\hat{x} \sqrt{s}}-e^{-\hat{y} \sqrt{s}}\right)\right)}\right), \\
& \Xi_{s, q ; 4}^{2}=\left(\frac{q}{s} \cdot \frac{\frac{e^{-x \sqrt{s}}-e^{-y \sqrt{s}}}{\sqrt{s}}+e^{-x \sqrt{s}}(x-a)-e^{-y \sqrt{s}}(y-a)+(b-a)\left(e^{-\tilde{y} \sqrt{s}}-e^{-\tilde{x} \sqrt{s}}\right)}{\frac{e^{-x \sqrt{q}}-e^{-y \sqrt{q}}}{\sqrt{q}}+e^{-x \sqrt{q}}(x-a)-e^{-y \sqrt{q}}(y-a)+(b-a)\left(e^{-\tilde{y} \sqrt{q}}-e^{-\tilde{x} \sqrt{q}}\right)}\right)^{\frac{1}{s-q}}, \\
& s \neq q, \\
& \Xi_{s, s ; 4}^{2}=\exp \left(\frac{y e^{-y \sqrt{s}}-x e^{-x \sqrt{s}}+\frac{3}{\sqrt{s}}\left(e^{-y \sqrt{s}}-e^{-x \sqrt{s}}\right)-e^{-x \sqrt{s}}(x-a)(2+x \sqrt{s})}{2 s\left(\frac{e^{-x \sqrt{s}}-e^{-y \sqrt{s}}}{\sqrt{s}}+e^{-x \sqrt{s}}(x-a)-e^{-y \sqrt{s}}(y-a)+(b-a)\left(e^{-\tilde{y} \sqrt{s}}-e^{-\tilde{x} \sqrt{s}}\right)\right)}\right),
\end{aligned}
$$

where $\hat{x}, \hat{y}, \tilde{x}$ and $\tilde{y}$ are the same as in (19). By using Theorem 2.6 , it is easy to see that

$$
M_{s, q}\left(x, \Phi_{i}, \Omega_{4}\right)=-(\sqrt{s}+\sqrt{q}) \log \mu_{s, q}\left(x, \Phi_{i}, \Omega_{4}\right), \quad i=1,2
$$

satisfy $\min \{a, b\} \leq M_{s, q}\left(x, \Phi_{i}, \Omega_{4}\right) \leq \max \{a, b\}$, showing that $M_{s, q}\left(x, \Phi_{i}, \Omega_{4}\right)(i=1,2)$ are means.

Remark 4.6 From (16), it is clear that $\mu_{s, q}\left(x, y, \Phi_{i}, \Omega\right)(i=1,2)$ for $\Omega=\Omega_{1}, \Omega_{2}, \Omega_{3}$ and $\Omega_{4}$ are monotonous functions in parameters $s$ and $q$.

\section{Competing interests}

The authors declare that they have no competing interests.

\section{Authors' contributions}

Both authors worked jointly on the results and they read and approved the final manuscript.

\section{Author details}

${ }^{1}$ Abdus Salam School of Mathematical Sciences, GC University, 68-B, New Muslim Town, Lahore 54600, Pakistan. ${ }^{2}$ Faculty of Textile Technology, University of Zagreb, Prilaz Baruna Filipovića 28A, 10000 Zegreb, Croatia.

\section{Acknowledgements}

The second author's research was supported by the Croatian Ministry of Science, Education and Sports, under the Research Grant 117-1170889-0888.

Received: 13 March 2012 Accepted: 21 June 2012 Published: 5 July 2012

\section{References}

1. Pečarić, JE, Proschan, F, Tong, YL: Convex Functions, Partial Orderings and Statistical Applications. Academic Press, New York (1992)

2. Dragomir, SS, Pearce, CEM: Selected Topics on Hermite-Hadamard Inequalities and Applications. RGMIA Monographs. Victoria University (2000). http://rgmia.vu.edu.au/monographs/.

3. Abramovich, S, Farid, G, Pečarić, J: More about Hermite-Hadamard inequalities, Cauchy's mean and superquadracity. J. Inequal. Appl. 2010, Article ID 102467 (2010). doi:10.1155/2010/102467

4. Pečarić, J, Roqia, G: Generalization of Stolarsky type means. J. Inequal. Appl. 2010, Article ID 720615 (2010). doi:10.1155/2010/720615 
5. Pečarić, J, Perić, J: Improvements of the Giaccardi and the Petrović inequality and related Stolarsky type means. An. Univ. Craiova, Ser. Mat. Inform. (2012, to appear)

6. Franjić, I, Khalid, S, Pečarić, J: On the refinements of the Jensen-Steffensen inequality. J. Inequal. Appl. 2011, 12 (2011)

7. Jakšetić, J, Pečarić, J, Rehman, AU: On Stolarsky and related means. Math. Inequal. Appl. 13(4), 899-909 (2010)

doi:10.1186/1029-242X-2012-155

Cite this article as: Khalid and Pečarić: On the refinements of the Hermite-Hadamard inequality. Journal of Inequalities and Applications 2012 2012:155.

Submit your manuscript to a SpringerOpen ${ }^{\odot}$ journal and benefit from:

- Convenient online submission

- Rigorous peer review

- Immediate publication on acceptance

- Open access: articles freely available online

- High visibility within the field

- Retaining the copyright to your article

Submit your next manuscript at $>$ springeropen.com 